\title{
A Proposed Internet of Everything Framework for Disease Prediction
}

\author{
https://doi.org/10.3991/ijoe.v15i04.9834
}

\author{
Noura E. Maghawry ( $\left.{ }^{\bowtie}\right)$, Samy Ghoniemy \\ The British University in Egypt, Cairo, Egypt \\ noura.elmaghawry@bue.edu.eg
}

\begin{abstract}
Social networks and Internet of things are two paradigms when integrated a new paradigm Internet of Everything is established that has its impact on revolutionizing various fields such as engineering, industry and healthcare. Social networks became nowadays of the most important web services on which people heavily rely, thus became a major source for information extraction for rational decision making considering individuals as social or socio sensors. Furthermore, people using sensors especially biological sensors enabled the use of internet of things technology in building intelligent healthcare systems. One of the challenges facing the design of such systems is the design of an intelligent recommender system that is able to deal with such big data. For that, this paper proposes a framework to develop an enhanced intelligent expert advisorbased health monitoring and disease awareness system. The proposed framework enables the researchers to design advisory systems that are able to observe physiological signals through the use of different biosensors and integrate it with historical medical data together with the massive data collected from social networks to provide accurate alerts and recommendations for many ailments inspected. The proposed Framework is designed to facilitate generic, dynamic and scalable process of integrating different types of social networks and biosensors.
\end{abstract}

Keywords - Internet of Things, Internet of Everything, biosensors, socio-sensors, Big data analytics, Healthcare.

\section{$1 \quad$ Introduction}

Online social networks dominated the majority of web services during the last decade, and researchers became more interested in relying on social networks for information extraction. The big data generated from both the heavy reliance on social networks, and data resulted from internet of things (IoTs) technologies make them very promising for valuable information extraction that could revolutionize various fields causing remarkable impact on the society.

The Internet of Things represents a paradigm where objects (things) are having capabilities of sensing, networking and processing allowing them to communicate and interact with one another or with other devices or services on the internet to achieve an objective [1]. 
The systems arising out from merging the two fields: social networks and IoTs allow the enrichment of both paradigms as the IoTs has its role on sensing the physical world whereas the social networks have its active contribution to the daily aspects of human world. Integrating IoTs with social networks composes a promising technology for adding the human experiences as sensors leading to highly intelligent services $[2,3]$.

The intersection between connections among people, together with the things, big data and smart processes, emphasizes the true power of a new paradigm called Internet of Everything (IoE) as shown in figure 1. IoE is expected to revolutionize various fields including marketing, investments, healthcare, education, military, and taking people through a new era of smart and intelligent services.

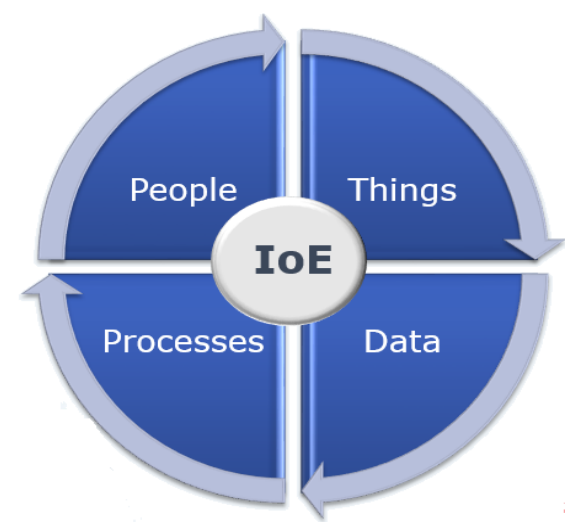

Fig. 1. The major components of Internet of Everything.

One of the important and challenging applications revolutionized by the Internet of Everything is building a smart, connected healthcare system as networked sensors, either worn on the body or embedded in our living environments, aiming to gather useful and relevant information for physical health.

Healthcare historically has been provided mainly in a reactive manner. With progress in the field of IoTs, the world has offered unique opportunities to better observe patients' physiological signals in order to provide healthcare in a more proactive manner. Adding human experiences supported by historical healthcare data ensure bringing personalized healthcare and intelligent monitoring systems to a challenging level.

To attain this intelligence level, it is essential to be able to analyze patient data in real time, and taking advantages of the social networks' big data of interest to provide quality of experience to the medical data and then turn it into reactive awareness to patients.

This is the major objective of this research. Thus, this paper presents four sections other than the introduction, section II presents the literature review and discusses similar related frameworks. The core research problem and the proposed solution methodology are discussed in section III. Section IV illustrates and presents the proposed Framework. Finally, section V presents the conclusion. 
IoTs paradigm aims at improving the quality of life for individuals, several frameworks for remote monitoring systems exist that use IoTs paradigm for healthcare monitoring, most of the frameworks proposed recently are designed for monitoring of elderly people, the following highlights the work where frameworks involving IoTs were proposed to serve healthcare recently. The research of Hassan Alieragh M. et al. was interested in reviewing the current state and future directions for integration of remote health monitoring technologies into the clinical practice of medicine and highlighted several of the challenges in sensing, analytics of data from wearable devices [4]. A study from user centered perspective highlighted in general the opportunities and challenges for IoTs in the future vision of healthcare remote monitoring systems, the research focused on studying IoTs enabled systems monitoring elderly people and trying to build a model that is user centric by taking into consideration the elderly requirements at the center of attention [5]. An IoTs framework was proposed for monitoring human biomedical signals in activities, these activities focuses on physical exertion using sensors from devices around the body area network of the user such as users' mobile environments [6]. An architecture of the Patient Health Monitoring System using IoTs devices is proposed to collect the medical parameters of a patient and evaluate them, the system notifies the individual with possible precautionary measures to be practiced by them [7]. Another IoT architecture proposed to be used in healthcare for monitoring the patients' ECG signals in an independent way, using a mobile tele-electrocardiograph [8].

On the other hand, there are studies published to illustrate the impact and the effect of social networks and communities on the healthcare, as an example the case studies presented to show the impact of social networking for raising the awareness of healthcare, and sensitizing individuals to their need for health care [9]. Other studies investigated the impact of social media on healthcare services and its applicability in Romania [10], and another study was held for investigating the impact of the evolution of Social Network Services in the healthcare domain and focused on specific healthoriented social networks such as Patients Like Me [11].

The recent frameworks focused on either enabling IoT paradigm for healthcare systems or investigating the beneficial impact of involving social networks in the healthcare field, which gives a solid background for building a framework, that involve both paradigms in one framework.

The major aim of this research is to develop an enhanced intelligent expert advisorbased health monitoring and disease awareness system; this will provide unique opportunities for better observing patients' physiological signals for reaching accurate alerts and recommendations for any ailments inspected. The research will involve adding human experiences supported by historical data background to the personalized healthcare intelligent monitoring systems.

The objective of the research is to build scalable and efficient techniques and algorithms over dynamically changing infrastructure on different levels, starting from data acquisition from different resources, data merging, integration, and filtration between different platforms, and ending with efficient classification and pattern matching techniques for reaching accurate alerts and recommendations for any ailments inspected. 
The system's knowledgebase will be built on medical healthcare facts and user experiences captured from information extracted by analyzing social networks' big data using big data analytics algorithms, in addition to relevant features extracted from processing sensors' data using real time processing techniques and algorithms.

\section{The Proposed Framework}

The proposed Framework is inspired from the fact that in the expected future, different technological revolutions in the fields of social networks, Internet of things will have its great impact on revolutionizing healthcare. The proposed framework in this research is as shown in figure 2.

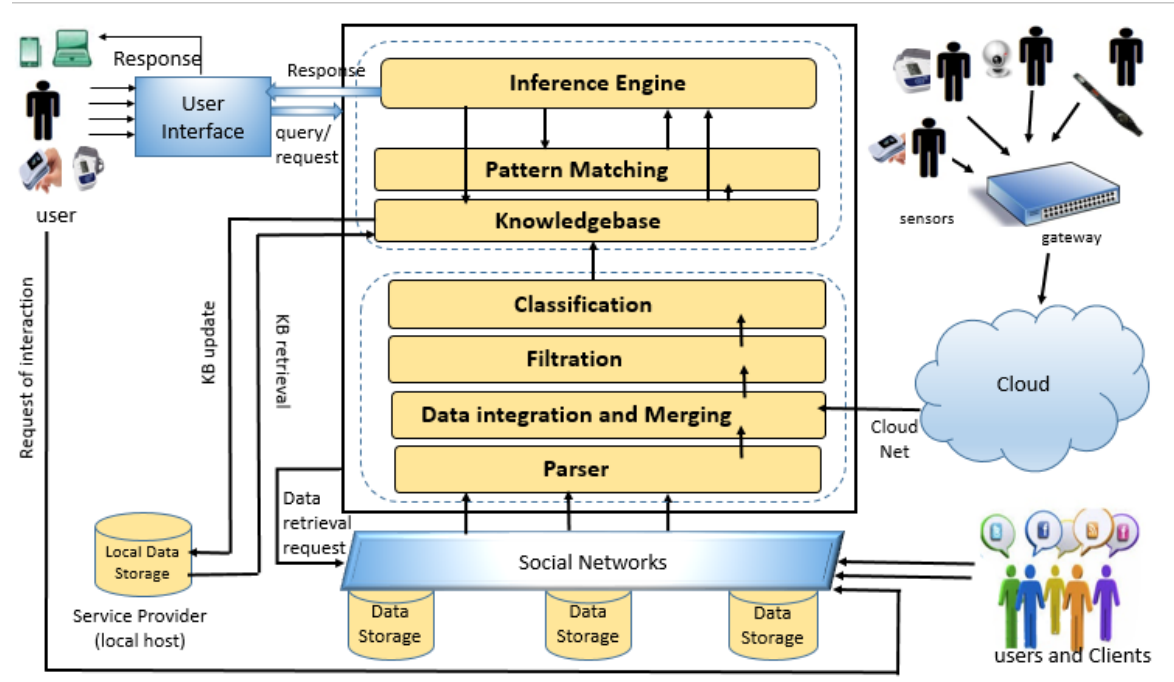

Fig. 2. The Proposed Framework.

The analytic platform adopted by the framework as shown in figure 3 is based on four stages: first stage will be information and feature extraction from unstructured resources from social networks' data and sensor data relevant to the disease in context, second stage will focus on building an improved focused crawler architecture that will adopt new techniques and algorithms for dealing with the information and feature extracted from the first stage, the third stage will be the Modeling stage where the model for mining the data for knowledge discovery will be built, and the fourth stage will build the predictive and awareness model thus building the intelligent expert system. 


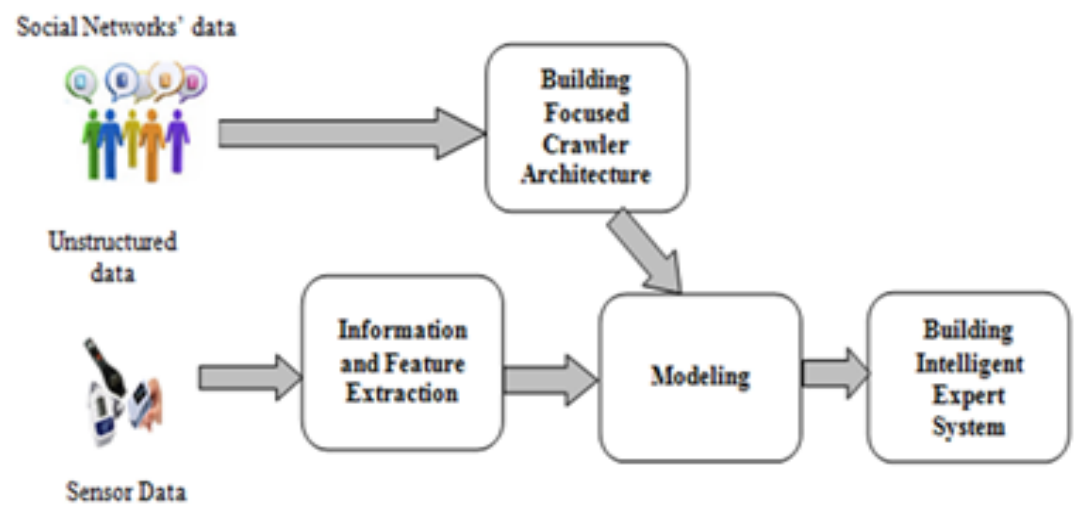

Fig. 3. Analytic platform adopted by the framework.

The first stage will benefit from gathering relevant sensor data through gateway available throughout the cloud and analyzing these data. The sensors' data gathered falls under the taxonomy of sensors stated in the work of Sow D. et al. used in medical informatics that involves physiological sensors measuring patient vital signs, wearable activity sensors measuring the gross of user activity that gives a wider aspect than the psychological sensors, contextual sensors embedded in the surrounding environment for measuring conditions like temperature and motion detection. The chain of processing sensor data as shown in figure 4 starts by sensor data collection for raw sensor data acquisition, filtration to filter data against outliers and noisy data, segmentation then feature extraction where the features relevant to the disease in context are extracted.

The second stage will implement a parser for topic detection and tracking for social networks, the parser will be capable of extracting information related to the disease in context, the parser will be the base for building an improved focused crawler architecture, which will adopt big data mining techniques and algorithms for finding documents relevant to symptoms and ailments for diseased patients. The focused crawler architecture will benefit the survey of Hetal J. et al .[13] that provides a reference for the previous work done for domain specific web crawling with the techniques and algorithms used and the parameters achieved, the survey studies the advantages and limitation of different algorithm of web crawling, that will help in creating a crawler overcoming the limitation of algorithms to improve the quality of crawler.

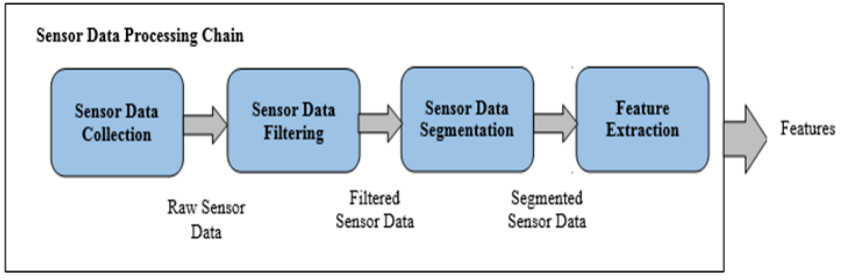

Fig. 4. Sensor data processing chain. 
The input to third stage - Modeling stage is the feature extracted from sensor data together with features and information extracted for social networks, the stage will face a challenge of implementing data integration and merging techniques between the information extracted from social networks, and the features extracted from sensor data, thus new reliable and robust techniques for data integration and merging will be investigated and applied, followed by filtration step where anomaly detection techniques will be implemented to identify statistically deviant data, followed by a classification model to classify the information and make it ready for the fourth stage.

The fourth stage is building the predictive and awareness model through building the intelligent expert system as shown in figure 5. The three basic components for the expert system are the inference engine, pattern matcher, and knowledge base. The repository formulating the knowledge base of the expert systems will be built from factual and heuristics knowledge formulated by user experiences gathered from social networks and sensor data together with historical medical healthcare. The inference engine is the software program that infers the requests from the user interface with the knowledge available in the knowledgebase by using the pattern matcher which will adopt efficient pattern matching techniques and algorithms and accordingly arouse alerts and awareness according to the matching level of the user with the patients' similarity.

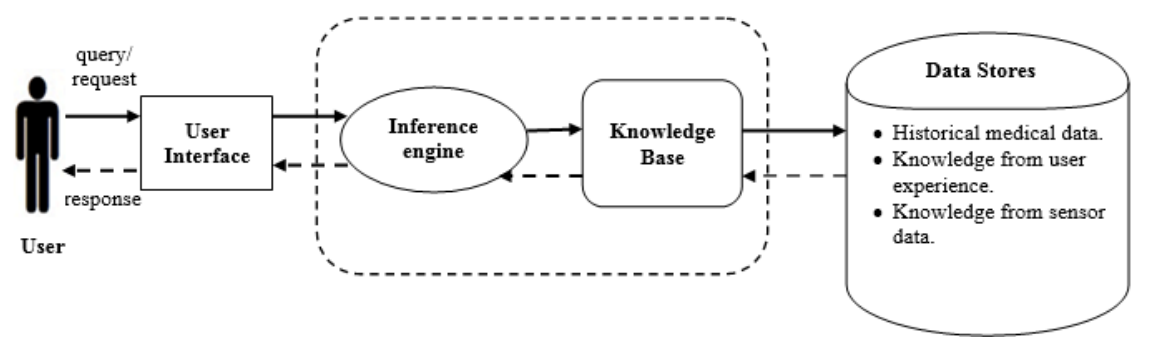

Fig. 5. The intelligent Expert System.

\section{Conclusion}

The proposed Framework serves as a promising framework in the Internet of Everything era for serving the healthcare field, the framework is a new trend if compared with other recent frameworks serving the healthcare field under the internet of things umbrella, new algorithms for data integration and merging for information extraction from both socio sensors and biosensors, followed by filtration and classification techniques for feeding the knowledge base of the advisory intelligent expert system taking into consideration the medical historical data will be a step to serve future applications in healthcare as it could be altered to serve different diseases. The framework is following the Internet of Everything paradigm where the paradigm is expected to have its great impact on revolutionizing healthcare. 
There are many challenges facing the framework, the heterogeneity nature of the data gathered from social networks and sensor data makes reaching robust algorithm a challenging task, reaching the relevant features serving the disease in context from both resources and applying the data integration and merging techniques will need building of an evaluation model that will be capable of measuring the accuracy of the predictive model.

\section{References}

[1] Whitmore A., Agarwal A., and Da Xu L., "The Internet of things-a survey of topics and trends", Information Systems Frontiers, vol. 17(2), pp. 261-274, April 2015. https://doi.org/10.1007/s10796-014-9489-2

[2] Ortiz A., Hussein D., Park A., Son N. Han and Crespi N., "The cluster between internet of things and social networks: Review and research challenges", Internet of Things Journal, IEEE, vol. 1(3), pp. 206-215, June 2014. https://doi.org/10.1109/JIOT.2014.2318835

[3] Atzori L., Iera A., Morabito G. and Nitti M., "The social internet of things (siot)-when social networks meet the internet of things: Concept, architecture and network characterization", Computer Networks, vol. 56 (16), pp. 3594-3608, November 2012. https://doi.org/1 0.1016/j.comnet.2012.07.010

[4] Hassanalieragh M. et al., "Health monitoring and management using Internet-of-Things (IoT) sensing with cloud-based processing: Opportunities and challenges" , Proceedings of IEEE International Conference on Services Computing, New York, NY, USA, pp. 285-292, July 2015.https://doi.org/10.1109/SCC.2015.47

[5] Azimi I., Rahmani AM., Liljeberg P., Tenhunen H., "Internet of things for remote elderly monitoring: A study from user-centered perspective", Journal of Ambient Intelligence and Humanized Computing, vol. 8 (2), pp: 1-17, April 2017. https://doi.org/10.1007/s12652016-0387-y

[6] Mora H., Gil D., Terol R.M., Azorín J., Szymanski J., "An IoT-Based Computational Framework for Healthcare Monitoring in Mobile Environments," Sensors 2017 Journal, the special issue Smart Sensor Networks and Technology for Healthcare Monitoring and Decision Making, vol. 17 (10), 2017.

[7] Arsand, E., Muzny M., Bradway M., Muzik J. and Hartvigsen G., "Performance of the first combined smartwatch and smartphone diabetes diary application study", Journal of Diabetes Science and Technology, vol. 9 (3), pp. 556-563, May 2015. https://doi.org/10. $1177 / 1932296814567708$

[8] Ungurean I., Brezulianu A., "An Internet of Things Framework for Remote Monitoring of the HealthCare Parameters", Advances in Electrical and Computer Engineering, vol.17(2), pp.11-16, May 2017. https://doi.org/10.4316/AECE.2017.02002

[9] Griffiths F. et al., "The Impact of Online Social Networks on Health and Health Systems: A Scoping Review and Case Studies." Policy \& Internet, vol. 7(4), pp. 473-496, September 2015 https://doi.org/10.1002/poi3.97

[10] Pentescu A., Cetină I., and Orzan G., "Social Media's Impact on Healthcare Services”, Proceedings of Economics and Finance, vol. 27, pp. 646-651, 2015. https://doi.org/10.1016/S2212-5671(15)01044-8

[11] Kotsilieris T., Pavlaki A., Christopoulou S. et al., "The impact of Social Networks on healthcare ", Social Network Analysis and Mining, vol. 7(18), December 2017. 
[12] Sow D., Turaga D.S., and Schmidt M., "Mining of Sensor Data in Healthcare: A Survey". In: Aggarwal C. editions Managing and Mining Sensor Data. Springer, Boston, MA, 2013. https://doi.org/10.1007/978-1-4614-6309-2_14

[13] Hetal J.Thankil, et al. "Domain Specific Web Crawler: A Survey." International Journal of Innovative Research in Science, Engineering and Technology, vol. 4 (12), pp. 2319-8753, 2015.

\section{Authors}

Noura E.Maghawry was born in Cairo, Egypt. She received her Bachelor from Faculty of Computer and Information Sciences, Ain Shams University and her master's degree from Arab Academy for science and technology and maritime transport. She is currently pursuing her Ph.D. degree while working as an Assistant Lecturer in the British University in Egypt.

Samy Ghoniemy received his Bachelor and Master of Engineering degrees in computer and communication engineering, in 1990 and 1996 respectively. He attended two years condensed program studying optoelectronic diploma in 1991. From 1991 to 2000 he was a Junior Lecturer and research assistant in MOD. He received his PhD degree from the Department of Systems and Computer Engineering, Carleton University, Ottawa, Canada. His primary research interests are, high performance computing, social networks analysis, deep learning for medical and healthcare applications, graph analytics, big data analytics, cloud computing, IoT, optical networks. AI, machine intelligence and cognitive science. He is currently a professor of computer networks and director of Advanced Informatics and Business Intelligence (AIBI) research center. He is a member of IEEE. S. Ghoniemy published more than 75 research papers in the field of computer engineering and computer science.

Article submitted 23 September 2018. Resubmitted 18 November 2018. Final acceptance 27 November 2018. Final version published as submitted by the authors. 